\title{
Fibromatosis colli
}

\author{
An infant with neck swelling
}

CPD

Larissa Passarello, Habib Bhurawala

\begin{abstract}
CASE
A girl aged 26 days with an enlarging neck lump was reviewed in a paediatric outpatient clinic. Examination revealed a firm and non-tender swelling on the right side of the neck. There was no overlying erythema. There was a mild torticollis without facial asymmetry. She was otherwise well. She was born at 41 weeks gestation via a vacuum delivery following induction of labour.
\end{abstract}

\section{QUESTION 1}

What is the differential diagnosis for this lump?

\section{QUESTION 2}

What investigations are indicated?

\section{ANSWER 1}

The conditions included in the differential diagnosis for a neck lump in infants are many and range from benign to sinister, including:

- fibromatosis colli

- branchial cleft cyst

- cervical lymphadenopathy

- lipoma

- lymphoma

- rhabdomyosarcoma

- neuroblastoma. ${ }^{1,2}$
Of these differentials, a branchial cleft cyst is the most common lateral congenital neck mass. ${ }^{3}$ Cervical lymphadenopathy can arise in children of all ages and is relatively common, caused predominately by bacterial or viral infections and rarely by malignancy. ${ }^{4}$ Despite the rarity of malignancy, it is worth noting that lymphoma is the most common cause of a malignant neck mass in children, and the most frequent site of origin of rhabdomyosarcoma is in the neck. ${ }^{3}$

\section{ANSWER 2}

The most appropriate first-line investigation is ultrasonography.

\section{CASE CONTINUED}

Ultrasonography of the neck found no abnormality in the left sternocleidomastoid muscle (Figure 1) but a fusiform area in the right sternocleidomastoid muscle measuring $20 \times 9 \times 19 \mathrm{~mm}$ (Figure 2). There was no internal vascularity, and some of the fibular structure of the muscle was preserved.

The infant was diagnosed with fibromatosis colli, and referred to a physiotherapist who prescribed exercises. At the six-week follow-up review, the lump was markedly reduced in size, and this was confirmed by ultrasonography.

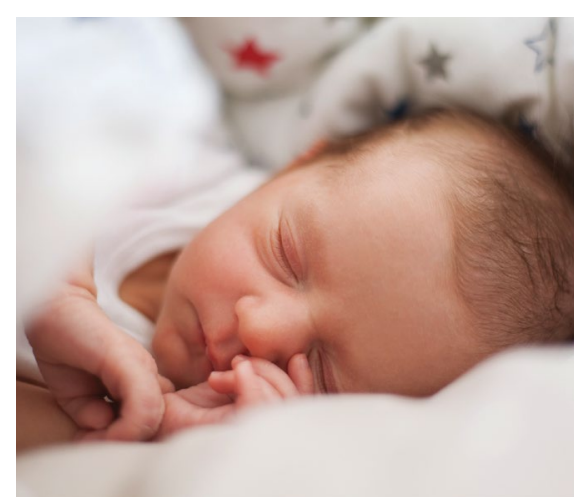

QUESTION 3

What is fibromatosis colli?

QUESTION 4

How is fibromatosis colli diagnosed?

QUESTION 5

What are the complications of fibromatosis colli?

QUESTION 6

What are the management options?

ANSWER 3

Fibromatosis colli is a benign mass of the sternocleidomastoid muscle, consisting of fibroblastic proliferation. ${ }^{5}$ It is also referred to as pseudotumour of the sternocleidomastoid muscle of infancy.

The reported prevalence is $0.4 \%$, and it affects boys more than girls. ${ }^{1}$ It is more common on the right side of the neck, is usually unilateral, and classically manifests as a firm, non-tender neck swelling that develops during the first four weeks of life..$^{2,6,7}$ Fibromatosis colli is associated with torticollis in approximately $20 \%$ of cases, and is the most common cause of congenital muscular torticollis. ${ }^{6,7}$

Although the pathogenesis is unclear, there is an association between fibromatosis colli and breech delivery, instrumental delivery and difficult labour. It has been postulated that in a difficult delivery, compression of the 
neck can cause ischaemic injury or venous occlusion, leading to damaged muscle fibres that then fibrose. ${ }^{6}$ Another proposed mechanism is tearing of the sternocleidomastoid during delivery resulting in a haematoma, ultimately causing atrophy of muscle fibres. ${ }^{8}$ When these lesions are biopsied, the most common feature on cytology is thin, spindle-shaped fibroblasts. ${ }^{5,7}$

\section{ANSWER 4}

Ultrasonography is the preferred imaging modality, with a sensitivity of $100 \%$. Ultrasonography is non-invasive, inexpensive and accessible. ${ }^{9}$ Features include a fusiform enlargement of the sternocleidomastoid muscle, generally in the lower two-thirds, with defined margins. Echogenicity varies, with $49 \%$ of masses being hyperechoic. ${ }^{6}$ Ultrasonography is also useful in excluding conditions considered in the differential diagnosis.

If further imaging is required to delineate the diagnosis, computed tomography (CT) or magnetic resonance imaging (MRI) can be used. CT images show isodense enlargement of the muscle, and on MRI

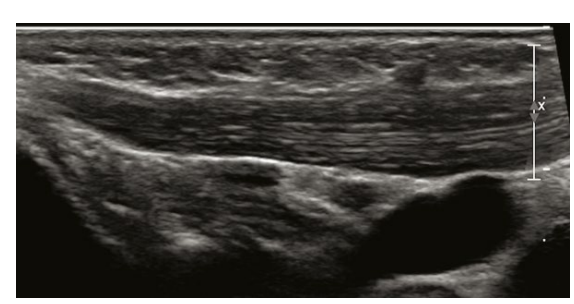

Figure 1. Longitudinal ultrasound image of the left sternocleidomastoid muscle (normal)

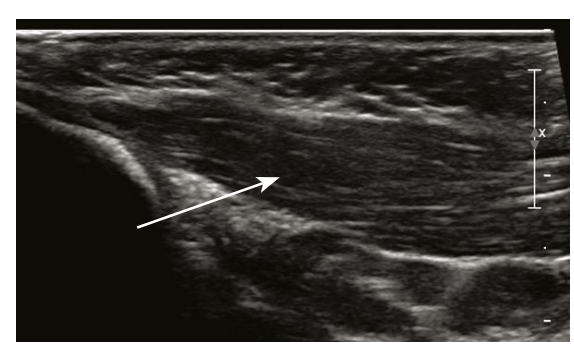

Figure 2. Longitudinal ultrasound image of the right sternocleidomastoid muscle; arrow indicates area of concern there is increased signal intensity on $\mathrm{T} 1$ when compared with $\mathrm{T} 2$ weighted images, demonstrating the presence of fibrous tissue. ${ }^{6,8}$ MRI can also provide information on the surrounding fascial planes, relationship to vascular structures and airway, and bony involvement. ${ }^{6}$

Fine needle aspiration for cytological investigation can be used to confirm diagnosis in a non-classical presentation. It is minimally invasive and can be used to avoid a more invasive procedure such as an excisional biopsy. ${ }^{7}$

\section{ANSWER 5}

Complications include:

- plagiocephaly

- facial asymmetry

- scoliosis

- permanent loss of neck mobility. ${ }^{9}$

\section{ANSWER 6}

Management is conservative and primarily involves physiotherapy with gentle, passive stretching exercise programs. ${ }^{8-10}$ Lee et al suggested a protocol of 15 manual stretches of the muscle sustained for one second, and 10 seconds between stretches, for three repetitions. This was performed three times per week. ${ }^{11}$ Active positioning at home can also be suggested, where parents are taught to encourage the infant to sleep with their head turned to the affected side. ${ }^{10}$ When commenced within four months of the onset of symptoms, resolution should occur within three to four months and in $90 \%$ of patients. ${ }^{9}$

For refractory cases, surgical tenotomy is an option. Botulinum toxin is currently being explored as an alternative treatment for children with ongoing symptoms despite physiotherapy, but further evidence of safety and long-term sequelae is required before this is accepted as a suitable treatment. ${ }^{9}$

\section{Key points}

- Fibromatosis colli is an unusual cause of a neck lump in infants, but the most common cause of congenital muscular torticollis.

- Diagnosis can be made confidently with a clinical examination and ultrasonography, allowing early parental reassurance and avoiding invasive investigations.

- The mainstay of treatment is physiotherapy, and results in resolution in the majority of cases.

\section{Authors}

Larissa Passarello MBBS, DCH, Paediatric Advanced Trainee, The Children's Hospital at Westmead, NSW. larissa.passarello@health.nsw.gov.au

Habib Bhurawala MBBS, MD, DCH, FRACP, Head of Paediatric Department, Nepean Hospital, NSW; Conjoint Lecturer, Sydney Medical School-Nepean, Discipline of Paediatrics, University of Sydney, NSW Competing interests: None.

Funding: None.

Provenance and peer review: Not commissioned, externally peer reviewed.

\section{References}

1. Oliveira JC, Abreu MS, Gomes FM. Sternocleidomastoid tumour in neonate: Fibromatosis colli. BMJ Case Rep 2018. doi: 10.1136/bcr-2017-223543.

2. Smiti S, Kulkarni NM, Singh J. Case report: Fibromatosis colli in a neonate. Indian J Radio Imaging 2010;20(1):45-46. doi: 10.4103/09713026.59753.

3. Smith A, Cronin M. Paediatric neck lumps: An approach for the primary physician. Aust J Gen Pract 2019;48(5):289-93.

4. Niedzielska G, Kotowski M, Niedzielski A, Dybiec E, Wieczorek P. Cervical lymphadenopathy in children - Incidence and diagnostic management. Int J Pediatr Otorhinolaryngol 2007;71(1):51-56. doi: 10.1016/j.ijporl.2006.08.024

5. Khalid S, Zaheer S, Wahab S, Siddiqui MA, Redhu N, Yusuf F. Fibromatosis colli: A case report. Oman Med J 2012;27(6):e011. doi: 10.5001/ omj.2012.126.

6. Ablin DS, Jain K, Howell L, West DC. Ultrasound and MR imaging of fibromatosis colli (sternomastoid tumor of infancy). Pediatr Radiol 1998;28(4):230-33. doi: 10.1007/s002470050337.

7. Baisakh MR, Mishra M, Narayanan R, Mohanty R. Cytodiagnosis of sternocleidomastoid tumor of infancy. J Cytol 2012;29(2):149-51. doi: 10.4103/0970-9371.97164.

8. Skelton E, Howlett D. Fibromatosis colli: The sternocleidomastoid pseudotumour of infancy. J Paediatr Child Health 2014;50(10):833-35. doi: 10.1111/jpc.12506

9. Tempark T, Chatproedprai S, Mahayosnond A, Wananukul S. Fibromatosis colli, overlooked cause of neonatal torticollis. Int J Pediatr Otorhinolaryngol 2012;7(1):15-17. doi: 10.1016/j. pedex.2011.07.007.

10. Cheng JCY, Tang SP, Chen TMK. Sternocleidomastoid pseudotumor and congenital muscular torticollis in infants: A prospective study of 510 cases. J Pediatr 1999;134(6):712-16. doi: 10.1016/S0022-3476(99)70286-6

11. Lee Y-T, Yoon K, Kim Y-B, et al. Clinical features and outcome of physiotherapy in early presenting congenital muscular torticollis with severe fibrosis on ultrasonography: A prospective study. J Pediatr Surg 2011;46(8):1526-31. doi: 10.1016/j. jpedsurg.2011.02.040. 